\title{
ON CENTRALIZERS OF INVOLUTIONS
}

MARCEL HERZOG

1. Introduction. The main purpose of this paper is to establish sufficient conditions for a group of even order to contain a normal elementary Abelian 2-subgroup of order at most 4 (Theorem 1). As a consequence it is shown that $\operatorname{PSL}(2,5)$ is the only simple group which contains an involution $x$ with the following property: the Sylow 2-subgroup of the centralizer $C$ of $x$ in $G$ is a noncyclic group of order 4 which is normal in $C$ (Theorem 3 ).

Several corollaries are derived from Theorem 1. In particular, a direct proof is given of the fact that $\operatorname{PSL}(2,5)$ is the only group which has no normal 2-complement, no normal elementary Abelian 2-subgroups of order less than 8 and which contains an involution with an elementary Abelian centralizer of order 4 (Theorem 2).

If $G$ is a group, $x \in G$ and $T$ is a subset of $G, C_{G}(x), \mathrm{Cl}_{G}(x), I(T)$, $o(T), o(x),\langle T\rangle, T^{\sharp}, Z(G)$ and $K(G)$ denote respectively: the centralizer of $x$ in $G$, the conjugate class of $x$ in $G$, the set of involutions in $T$, the number of elements in $T$, the order of $x$, the group generated by $T, T-\{1\}$, the center of $G$ and the largest normal subgroup of $G$ of odd order. If $P$ is a $p$-group then $\Omega_{1}(P)$ is the subgroup of $P$ generated by elements of $P$ of order $p$.

From now on $G$ will be a group of even order, $x$ a fixed involution of $G, K=K(G), C=C_{G}(x), I=I\left(C_{G}(x)\right), \mathrm{Cl}(x)=\mathrm{Cl}_{G}(x)$, and $S$ a fixed Sylow 2-subgroup of $G$ containing $x$ such that $S_{0}=S \cap C=$ Sylow 2 -subgroup of $C$. We are ready to state the results.

Theorem 1. Suppose that there exists $y \in I-\mathrm{Cl}(x)$ such that

$$
C_{G}(u) \cap \mathrm{Cl}_{G}(y) \subset C_{G}(y)
$$

for all $u \in I$. Then $\left\langle\mathrm{Cl}_{G}(y)\right\rangle$ is a proper elementary Abelian normal 2-subgroup of $G$.

If, in addition, $I \cap\left\langle\mathrm{Cl}_{G}(y)\right\rangle=\{y\}$, then $o\left(\left\langle\mathrm{Cl}_{G}(y)\right\rangle\right) \leqq 4$.

Corollary 1. Suppose that the following conditions hold:

(a) $I=I\left(C_{G}(u)\right)$ for all $u \in \mathrm{Cl}(x) \cap I$;

(b) $I\left(C_{G}(y)\right)=I\left(C_{G}(z)\right)$ for all $y, z \in I-\mathrm{Cl}(x)$. Then one of the following statements holds.

(i) $G$ has one class of involutions and $\langle I\rangle$ is an elementary Abelian normal 2-subgroup of $C$.

Received by the editors April 19, 1968 and, in revised form, June 17, 1968. 
(ii) $G$ has at least two classes of involutions and it contains a proper elementary Abelian normal 2-subgroup.

Corollary 2. Suppose that $o(I) \leqq 3$. Then one of the following statements holds.

(i) $S_{0}=S, x$ is the only involution in $S$ and $\langle x\rangle K$ is a normal subgroup of $G$.

(ii) $S_{0}=S, S$ contains exactly 3 involutions and $\langle x\rangle K$ is a proper normal subgroup of $G$.

(iii) $S_{0}=S, G$ has one conjugate class of involutions.

(iv) $G$ has at least 2 classes of involutions and it contains a normal elementary Abelian subgroup of order at most 4.

Corollary 2 immediately yields

Corollary 3. Suppose that $o(I) \leqq 3$ and $G$ is simple. Then $S=S_{0}$ and $G$ has only one conjugate class of involutions.

In case that $C$ is elementary Abelian of order 4 we get the following

Theorem 2. Suppose that $C=\{1, x, y, x y\}$ is elementary Abelian and $G$ has neither a normal 2-complement nor a normal elementary Abelian 2-subgroup of order less than 8 . Then $G \cong \operatorname{PSL}(2,5)$.

The following corollary is an easy consequence of Theorem 2, the results of Suzuki in [6] and the results of Feit and Thompson in [2].

COROLlaRY 4. Let $G$ be a finite noncyclic simple group containing an element $w$ such that $o\left(C_{G}(w)\right) \leqq 4$. Then $G$ is isomorphic to one of the following groups: $\operatorname{PSL}(2,5), \operatorname{PSL}(2,7), A_{6}$ and $A_{7}$.

Our final theorem requires the deep results of Gorenstein and Walter [5] with respect to groups with a dihedral Sylow subgroup of order 4.

Theorem 3. Suppose that $S_{0}=\{1, x, y, x y\}$ is elementary Abelian, $S_{0}$ is normal in $C$ and $G$ is simple. Then $G \cong \operatorname{PSL}(2,5)$.

The proof of Theorem 1 utilizes the following lemma, which is of independent interest.

LEMma. Let $U$ be a subgroup of the group $H$ and let $w$ be an involution of $H$ which normalizes $U$ leaving fixed exactly two elements of $U, 1$ and $y$. Let $V$ be a normal, w-invariant noncyclic elementary Abelian subgroup of $U$ containing $y$. Then $V$ is a Sylow 2-subgroup of $U, o(V)=4$, and $U / V$ is Abelian. 
2. Proof of the Lemma, Theorem 1 and Corollary 1. We begin with the proof of the Lemma. Obviously $y$ is an involution. First assume that $o(V)=4, V=\{1, y, z, y z\}$; then $z^{w}=y z$. Suppose that $U / V$ is not an Abelian group of odd order. Then $w$ fixes an element of $(U / V) \boldsymbol{t}$, say $u V$. Thus one of the following holds:

$$
\begin{aligned}
& u^{w}=u y \quad \text { and } \quad u=u^{w^{2}}=u \\
& =u z \quad=u y \\
& =u y z \quad=u y .
\end{aligned}
$$

Hence we must have $u^{w}=u y$; but then $(u z)^{w}=(u y)(y z)=u z$ a contradiction. Thus $U / V$ is an Abelian group of odd order. If $o(V)>4$, then $w$ fixes an element of $(V /\langle y\rangle)^{*}$, say $z\langle y\rangle$, and $V_{0}=\langle z, y\rangle$ is a normal, $w$-invariant, elementary Abelian subgroup of $V$ containing $y, o\left(V_{0}\right)$ $=4$, and by the first part $V=V_{0}$, a contradiction. The proof of the Lemma is complete.

To prove Theorem 1, suppose first that $\mathrm{Cl}_{G}(y) \nsubseteq C_{G}(y)$ and let $t \in \mathrm{Cl}_{G}(y)-C_{G}(y)$. By a result of Brauer and Fowler [1, p. 572], there exists $w \in I(G)$ such that $w \in I\left(C_{G}(x)\right) \cap C_{G}(t) \subset I$. Hence by $\left(^{*}\right)$ $t \in C_{G}(w) \cap \mathrm{Cl}_{G}(y) \subset C_{G}(y)$ a contradiction. It follows that $\mathrm{Cl}_{G}(y)$ $\subset C_{G}(y)$ and $\left\langle\mathrm{Cl}_{G}(y)\right\rangle=H$ is a normal subgroup of $G$ contained in $C_{G}(y)$. If $C_{G}(y)=G$, then $H=\langle y\rangle \neq G$ and the theorem follows. If $C_{G}(y) \neq G$, then $H$ is a proper normal subgroup of $G$ and obviously $y \in \Omega_{1}(P) \triangleleft G$ where $P$ is the Sylow 2-subgroup of $Z(H)$. Hence $\mathrm{Cl}_{G}(y) \subset \Omega_{1}(P)$ and $H$ is elementary Abelian. Finally suppose that $o(H) \geqq 8$ and $I \cap H=\{y\}$. Then $x$ leaves only $y$ and 1 fixed in $H$ and by the Lemma $o(H)=4$, a contradiction. Thus $o(H) \leqq 4$ and the proof of Theorem 1 is complete.

It remains to prove Corollary 1 . If $I \subset \mathrm{Cl}(x)$, then each element of $I$ belongs to the center of some Sylow 2-subgroup of $G$ and therefore $G$ has one class of involutions. By (a), $\langle I\rangle$ is an elementary Abelian normal 2-subgroup of $C$ and (i) holds. Suppose finally that $I \nsubseteq \mathrm{Cl}(x)$ and let $y \in I-\mathrm{Cl}(x)$. It follows from (b) that the elements of $I-\mathrm{Cl}(x)$ commute with each other. Thus for all $u \in I \cap \mathrm{Cl}(x)$,

$$
C_{G}(u) \cap \mathrm{Cl}_{G}(y)=I \cap \mathrm{Cl}_{G}(y) \subset C_{G}(y),
$$

and for all $u \in I-\mathrm{Cl}(x)$,

$$
C_{G}(u) \cap \mathrm{Cl}_{G}(y)=I\left(C_{G}(y)\right) \cap \mathrm{Cl}_{G}(y) \subset C_{G}(y) .
$$

It follows then by Theorem 1 that $G$ has a proper normal elementary Abelian 2-subgroup. 
3. Proof of Theorem 2 and Corollaries 2 and 4. We begin with Corollary 2. If $o(I)=1$, then $S_{0}=S, x$ is the only involution in $S$ and by [3], $\langle x\rangle K$ is a normal subgroup of $G$, as described in (i). As $o(I) \neq 2$, let $o(I)=3, I=\{x, y, x y\}$. If no element of $I$ is conjugate to $x$ in $G$, then $N_{S}\left(S_{0}\right)=S_{0}, S=S_{0}$, and by [3] $\langle x\rangle K \triangleleft G$. Since $o(I)=3,\langle x\rangle K \neq G$ and (ii) holds. If all the elements of $I$ are conjugate in $G$, then again $S_{0}=S$ and (iii) holds. Suppose finally that $x$ is conjugate to $x y$ in $G$, but not to $y$. Then $I\left(C_{G}(x y)\right)=I$ and by Corollary $1,\left\langle\mathrm{Cl}_{G}(y)\right\rangle$ is a normal elementary Abelian 2-subgroup of $G$. Hence, as either $\left\langle\mathrm{Cl}_{G}(y)\right\rangle$ $=\langle y\rangle$ or $\mathrm{Cl}_{G}(y)$ contains an element which does not commute with $x$, $I \cap\left\langle\mathrm{Cl}_{G}(y)\right\rangle=\{y\}$ and by Theorem $1, o\left(\left\langle\mathrm{Cl}_{G}(y)\right\rangle\right) \leqq 4$, so that (iv) holds. This completes the proof of Corollary 2.

We continue with Theorem 2 . If $C=S$, then by Lemma 15.2.4 of [4], $G$ has only one class of involutions and $N=N_{G}(C) \cong \operatorname{PSL}(2,3)$. Thus $C$ contains the centralizer of each of its nonunit elements and by Theorem 9.3.2 in [4], due to Suzuki, $G$ is a Zassenhaus group of degree 5 with $N$ the subgroup fixing a letter. Thus $N$ is a Frobenius group with complement of order $e=3$ and kernel of order $n=4$. Since $e$ is odd and $e=n-1$, it follows from Theorems 13.3.5 and 13.1.1 in [4], due to Zassenhaus, that $G \cong \operatorname{PSL}(2,4) \cong \operatorname{PSL}(2,5)$. Next assume that $C \neq S$ and let $y \in C \cap Z(S)$. As $N_{S}(C) \neq C, x y$ is conjugate to $x$ in $G$ and $C_{G}(x y)=C$. Since $y$ is not conjugate to $x$ in $G$, it follows from Theorem 1 that $\left\langle\mathrm{Cl}_{G}(y)\right\rangle$ is a normal elementary Abelian 2-subgroup of $G$. As before $I \cap\left\langle\mathrm{Cl}_{G}(y)\right\rangle=\{y\}$, and it follows by Theorem 1 that $o\left(\left\langle\mathrm{Cl}_{G}(y)\right\rangle\right) \leqq 4$ in contradiction to our assumptions. The proof is complete.

It remains to prove Corollary 4. If $o\left(C_{G}(w)\right)=2$, then $G$ is not simple. If $o\left(C_{G}(w)\right)=3$, then by [2], $G$ is isomorphic either to $\operatorname{PSL}(2,5)$ or to $\operatorname{PSL}(2,7)$. If $o\left(C_{G}(w)\right)=4$ and $o(w)=4$, then by [6], $G$ is isomorphic to one of the groups $\operatorname{PSL}(2,7), A_{6}$ and $A_{7}$. If, finally, $o\left(C_{G}(w)\right)=4$ and $o(w)=2$, then by Theorem $2, G \cong \operatorname{PSL}(2,5)$.

4. Proof of Theorem 3. If $S=S_{0}$, then by [5], $G \cong \operatorname{PSL}(2, q), q>3$. If $q$ is even, then $G \cong \operatorname{PSL}(2,4) \cong \operatorname{PSL}(2,5)$. If $q$ is odd, then the centralizer $C$ of an involution of $G$ is a dihedral group of order $q+\epsilon$, $\epsilon= \pm 1$. For $S$ to be normal in $C, q+\epsilon=4$ and $q=5$. Thus again $G$ $\cong \operatorname{PSL}(2,5)$. Suppose next that $S_{0} \neq S,\{y\}=Z(S) \cap S_{0}{ }^{*}$. Then $N_{S}\left(S_{0}\right) \neq S_{0}, x y$ is conjugate to $x$ in $G$ and $S_{0}$ is the normal Sylow 2-subgroup of $C_{G}(x y)$. As $y$ is not conjugate to $x$ in $G$, it follows from Corollary 1 that $G$ contains a proper, nontrivial, normal subgroup, in contradiction to the simplicity of $G$. The proof is complete. 


\section{REFERENCES}

1. R. Brauer and K. A. Fowler, On groups of even order, Ann. of Math. 44 (1943), 57-79.

2. W. Feit and J. G. Thompson, Finite groups which contain a self-centralizing subgroup of order 3, Nagoya Math. J. 21 (1962), 185-197.

3. G. Glauberman, Central elements in core-free groups, J. Algebra 4 (1966), 403-420.

4. D. Gorenstein, Finite groups, Harper and Row, New York, 1968.

5. D. Gorenstein and J. H. Walter, The characterization of finite groups with dihedral Sylow 2-subgroups. I, II, III, J. Algebra 2 (1965), 85-151, 218-270, 334-393.

6. M. Suzuki, On finite groups containing an element of order four which commutes only with its powers, Illinois J. Math. 3 (1959), 255-271.

Untversity of California, Santa Barbara 